analog items made parents feel are expected to be related to parents' empathic concern. Finally, parents' responses to the analog tasks are anticipated to be strongly associated with parents' self-reported parental empathy. DISCUSSION/SIGNIFICANCE OF IMPACT: Valid, novel assessments of parental empathy can impact the parenting literature as well as community intervention and prevention efforts with parents. Such analog tasks can bolster parenting research but they may also be translated to the community setting as a training tool wherein parents are taught new skills that promote more positive parenting.

4030

\section{Development of a Falls-Prevention Self-Management Plan for Community Dwelling Older Adults}

Jennifer L. Vincenzo ${ }^{1}$, Leanne L. Lefler ${ }^{1}$, Susan Kane Patton ${ }^{2}$, and Jeanne $\mathrm{Wei}^{3}$

${ }^{1}$ University of Arkansas Translational Research Institute; ${ }^{2}$ University of Arkansas; ${ }^{3}$ University of Arkansas for Medical Sciences

OBJECTIVES/GOALS: 1 . To determine older adults' opinions on content that is valuable for inclusion in a falls-prevention self-management plan. 2. To determine older adults' recommendations of mode(s) to promote adherence to falls prevention recommendations. METHODS/STUDY POPULATION: On-on-one semi structured interviews with older adults are ongoing to determine their opinions on content for inclusion in a falls-prevention self-management plan and recommendations for mode of delivery. As in our prior investigations, we used the theoretical constructs of the health belief model to develop our questions. Interviews will be recorded and transcribed. Data will be entered into MAXQDA12 and coded. Concurrent data collection and analysis will continue until theoretical saturation of themes are achieved. Through this iterative process, we will identify content and mode of delivery for a falls- prevention self-management plan for implementation with older adults. RESULTS/ANTICIPATED RESULTS: We anticipate we will have conducted enough interviews to achieve data saturation by February, 2020. We expect the results of this qualitative investigation to guide the development of a falls-prevention self-management plan that includes targeted implementation and adherence strategies deemed acceptable and feasible for use among older adults following community-based falls-risk screenings. DISCUSSION/SIGNIFICANCE OF IMPACT: Falls are the leading cause of morbidity and mortality among older adults. Adherence to falls prevention among older adults is poor, even among those that voluntarily seek out recommendations. The results of this study will assist with development and pilot testing of a falls-prevention self-management plan to assist older adults to adhere to recommendations.

Development of a Patient Registry and Biospecimen Repository to Identify Biomarkers in Nontuberculous Mycobacteria Lung Disease

Bryan Garcia ${ }^{1}$, Abigail Grady ${ }^{2}$, Lilian Christon², Patrick Flume ${ }^{2}$, and Susan Dorman²

${ }^{1}$ Medical University of South Carolina; ${ }^{2}$ MUSC

OBJECTIVES/GOALS: Non-tuberculous mycobacteria (NTM) is an emerging infection in the United States. Therapeutic development is impaired in NTM due to lack of biomarkers associated with disease activity and treatment response. To address this, we established a cross-sectional patient registry and biorepository from NTM patients. METHODS/STUDY POPULATION: Beginning August 2019 patients were recruited in a cross-sectional format from the bronchiectasis and NTM clinics at our institution. All patients provided at least one sputum sample in the six months prior to inclusion. Clinical and epidemiologically relevant data was obtained, patient reported outcome measures including the SGRQ were provided, and blood specimens were processed and preserved. Patients were grouped based on clinical phenotype and descriptive statistics were reported as means and standard deviations. Serum inflammatory profiles will be analyzed using standard Luminex assays. RESULTS/ANTICIPATED RESULTS: 72 patients with prior NTM isolation from sputum have been recruited including 28 patients that do not meet ATS guidelines for NTM treatment (colonized), 29 patients are currently receiving treatment, and 15 patients that have a history of completing therapy. Among all NTM patients, the mean age was $59.5 \pm 17.6$ years and $80.8 \%$ were female. The mean $\mathrm{FEV}_{1}$ percent predicted among these patients was $68.0 \pm 21.5 \%$ and mean BMI was $22.35 \pm 4.1$. The most common mycobacterial species isolated was Mycobacterium Avium Complex (47\%). The mean SGRQ total score was $40.2 \pm 20.3$ among all NTM patients. We plan to perform standard Luminex assays to identify inflammatory profiles associated with disease phenotype. DISCUSSION/ SIGNIFICANCE OF IMPACT: We developed a cross sectional cohort of NTM patients including a registry of clinically relevant data for phenotyping and an accompanying biospecimen repository. Our long-term goal is to identify biomarkers indicative of disease activity and treatment response using these components.

4284

Development of a Survey Instrument to Predict Uptake of and Adherence to Active Surveillance among Men with Low-Risk Prostate Cancer

Aaron T Seaman ${ }^{1}$, Kathryn L. Taylor, Kimberly Davis, Kenneth G. Nepple, Michelle A. Mengeling, Heather Schacht Reisinger, and Richard M. Hoffman

${ }^{1}$ University of Iowa Institute for Clinical and Translational Science

OBJECTIVES/GOALS: Active surveillance (AS) is a recognized strategy to manage low-risk prostate cancer (PCa) in the absence of cancer progression. Little prospective data exists on the decisional factors associated with selecting and adhering to AS in the absence of cancer progression. We developed a survey instrument to predict AS uptake and adherence. METHODS/STUDY POPULATION: We utilized a three-step process to develop and refine a survey instrument designed to predict AS uptake and adherence among men with low-risk PCa: 1) We identified relevant conceptual domains based on prior research and a literature review. 2) We conducted 21 semistructured concept elicitation interviews to identify patient-perceived barriers and facilitators to AS uptake and adherence among men with a low-risk PCa who had been on AS for $\geq 1$ year. The identified concepts became the basis of our draft survey instrument. 3) We conducted two rounds of cognitive interviews with men with low-risk PCa $(\mathrm{n}=12 ; \mathrm{n}=6)$ to refine and initially validate the instrument. RESULTS/ANTICIPATED RESULTS: Relevant concepts identified from the initial interviews included the importance of patient: knowledge of their PCa risk, value in delaying treatment, trust in urologist and the AS surveillance protocol, and perceived social support. Initially, the survey was drafted as a single instrument to be administered after a patient had selected AS comprising sections on patient health, AS selection, and AS adherence. Based on the first round of cognitive interviews, we revised the single 
instrument into two surveys to track shifts in patient preference and experience. The first, administered at diagnosis, focuses on selection, and the second, a 6-month follow up, focuses on adherence. Following revisions, participants indicated the revised 2-part instrument was clear and not burdensome to complete. DISCUSSION/ SIGNIFICANCE OF IMPACT: The instrument's content validity was evaluated through cognitive interviews, which supported that the survey items' intended and understood meanings were isomorphic. In the next phase, we plan to conduct a large-scale prospective cohort study to evaluate the predictive validity, after which it will be available for public research use.

\section{7}

Discrimination, Social Cohesion, and Hypertension: A Cross-Sectional Analysis from the REGARDS Study

Zachary H McCann ${ }^{1}$, April Carson, Verna Keith, Timothy Plante, Rachel Stuckwisch, and Suzanne Judd

${ }^{1}$ University of Alabama at Birmingham

OBJECTIVES/GOALS: This research has two goals. The first is to establish the relationship between hypertension, discrimination, and social cohesion in the general populaiton. The hypotheses for these relationships are:

- Hypothesis 1: Self-reported discrimination will be positively associated with incidence of hypertension in all REGARDS participants.

- Hypothesis 2: Self-reported neighborhood cohesion will attenuate the effects of discrimination on the development of hypertension.

- Hypothesis 3: Increased levels of Self-reported social cohesion will moderate the effects of discrimination on the likelihood of being hypertensive by weakening the relationship between discrimination and the likelihood of being hypertensive.

The second goal of this research is examining the the nexus of race and social cohesion, to understand if racial effects exist. The hypotheses for this goal are:

- Hypothesis 4: Self-reported discrimination will be positively associated with prevalent hypertension in black REGARDS participants over and above the association between discrimination and hypertension in white REGARDS participants.

- Hypothesis 5: Increased levels of self-reported social cohesion for will not moderate the effects of discrimination on the likelihood of being hypertensive by weakening the relationship between discrimination and the likelihood of being hypertensive as strongly for black REGARDS participants as it does for white REGARDS participants

METHODS/STUDY POPULATION: The population under investigation will be respondents to The Reasons for Geographic and Racial Differences in Stroke (REGARDS) study at UAB. REGARDS participants are 45+ years old, and come from across the United States. The study will use the second wave of in-home data to assess relationships between discrimination, hypertension, and social cohesion. First we will calculate descriptive statistics (means and standard deviations or $\mathrm{N}$ and \%) for each of the main variables and covariates. Modified Poisson regression will be used to model the association between discrimination and likelihood of hypertension, as well as the moderating effects of neighborhood social cohesion. We will run additional modified Poisson models including 1) demographic covariates (age, gender, race, rurality, education), 2) demographic covariates plus lifestyle factors, such as smoking status, BMI, exercise frequency, and 3) demographic covariates, lifestyle factors, and social characteristics, including insurance status, social support, getting around. To understand the main effect of social cohesion on hypertension, we will run a second set of models, following the same series of steps. A final series of models will test the interaction between perceived discrimination*perceived neighborhood cohesion. This model will be followed by the same series of steps as the global hypertension models. If the interaction is significant, postestimation will be used to model how different levels of perceived discrimination and cohesion are expected to interact to affect the likelihood of hypertension. If any of the covariates violates assumptions about distributions, data transformations will be explored. After conducting an analysis on the effects of discrimination on hypertension, and the buffering effect of social cohesion on the full sample population, the sample will be stratified by race so that the associations between discrimination, social cohesion, hypertension, and race are fully explored, consistent with previous literature. The stratified samples will be run through the same series of models as the full sample, providing information on how race, hypertension, and social cohesion are associated in and unadjusted models, models adjusted for demographic characteristics, models adjusted for sociodemographic and lifestyle characteristics, and finally full models that adjust for sociodemographic, lifestyle, and social characteristics. These models will compare each of these sets of characteristics, as well as the interaction between perceived discrimination* perceived neighborhood cohesion for stratified samples. This will allow researchers to compare the effects of discrimination, and the buffering effect of neighborhood cohesion across models. RESULTS/ANTICIPATED RESULTS: We anticipate that in a general population, social cohesion will attenuate the positive association of discrimination and hypertension. For blacks, however, higher levels of lifetime discrimination as well as lower levels of social cohesion in primarily black neighborhoods are expected to lead to both higher rats of hypertension and a lower degree of attenuation of the relationship between discrimination and hypertension. DISCUSSION/SIGNIFICANCE OF IMPACT: This study will help to elucidate the social nature of hypertension. By examining how perceptions of discrimination and social cohesion are associated with hypertension, interventions directed at improving heart health will be able to be effectively implemented at the community level by targeting specific social factors that can improve health outcomes. In addition, this research will provide insight into groups that may be particularly vulnerable to hypertension, advancing both the sociological and cardiology literature as it relates to discrimination, social cohesion, race and health.

4378

Dynamic Influences on Population Health Management by Asthma Community Health Worker (CHW) Programs: An Agent Based Modeling Approach*

Bradley Kramer ${ }^{1}$, Jessica Jones-Smith ${ }^{1}$, and Bryan Weiner ${ }^{1}$

${ }^{1}$ University of Washington

OBJECTIVES/GOALS: Twenty years of evidence show CHW homevisits for asthma improve population health and lower overall health care system costs by reducing costly Emergency Department (ED) 